PROCEEDINGS OF THE

AMERICAN MATHEMATICAL SOCIETY

Volume 134, Number 12, December 2006, Pages 3649-3651

S 0002-9939(06)08448-6

Article electronically published on June 29, 2006

\title{
ALMOST SIMPLE GROUPS OF SUZUKI TYPE ACTING ON POLYTOPES
}

\author{
DIMITRI LEEMANS
}

(Communicated by John R. Stembridge)

\begin{abstract}
Let $S=S z(q)$, with $q \neq 2$ an odd power of two. For each almost simple group $G$ such that $S<G \leq A u t(S)$, we prove that $G$ is not a C-group and therefore is not the automorphism group of an abstract regular polytope. For $G=S z(q)$, we show that there is always at least one abstract regular polytope $\mathcal{P}$ such that $G=\operatorname{Aut}(\mathcal{P})$. Moreover, if $\mathcal{P}$ is an abstract regular polytope such that $G=\operatorname{Aut}(\mathcal{P})$, then $\mathcal{P}$ is a polyhedron.
\end{abstract}

\section{INTRODUCTION}

In 1, Leemans and Vauthier built an atlas of abstract regular polytopes for small groups. The groups $S z(8)$ and $\operatorname{Aut}(S z(8))=S z(8): 3$ were among the groups analysed by Leemans and Vauthier. It turns out that $S z(8)$ has seven polytopes, all of rank three, and that $\operatorname{Aut}(S z(8))$ has no polytopes.

In this short note, we prove that if $G=S z(q)$ with $q \neq 2$ an odd power of 2, then all the abstract regular polytopes having $G$ as automorphism group are of rank three (and there exists at least one such polytope for each value of $q$ ). Moreover, if $S z(q)<G \leq \operatorname{Aut}(S z(q))$, we show that $G$ is not a C-group and therefore that there cannot exist an abstract regular polytope having $G$ as automorphism group.

\section{Premilinaries}

Thin regular residually connected geometries with a linear diagram, abstract polytopes and string C-groups are the same mathematical objects. The link between these objects may be found for instance in [2. Here we take the viewpoint of string C-groups because it is the easiest and the most efficient one to define abstract regular polytopes.

As defined for instance in 2, a C-group is a group $G$ generated by pairwise distinct involutions $\rho_{0}, \ldots, \rho_{n-1}$ which satisfy the following property, called the intersection property:

$$
\forall J, K \subseteq\{0, \ldots, n-1\},\left\langle\rho_{j} \mid j \in J\right\rangle \cap\left\langle\rho_{k} \mid k \in K\right\rangle=\left\langle\rho_{j} \mid j \in J \cap K\right\rangle .
$$

Received by the editors June 24, 2005 and, in revised form, August 1, 2005.

2000 Mathematics Subject Classification. Primary 52B11; Secondary 20 D06.

Key words and phrases. String C-groups, abstract regular polytopes, thin regular geometries, Suzuki simple groups.

(C)2006 American Mathematical Society Reverts to public domain 28 years from publication 
A C-group $\left(G,\left\{\rho_{0}, \ldots, \rho_{n-1}\right\}\right)$ is a string C-group if its generators satisfy the following relations:

$$
\left(\rho_{j} \rho_{k}\right)^{2}=1_{G} \forall j, k \in\{0, \ldots, n-1\} \text { with }|j-k| \geq 2 .
$$

In this short note, we prove the following result.

Theorem 1. Let $S z(q) \leq G \leq \operatorname{Aut}(S z(q))$ with $q=2^{2 e+1}$ and $e>0$ a positive integer. Then $G$ is a $C$-group if and only if $G=S z(q)$. Moreover, if $\left(G,\left\{\rho_{0}, \ldots, \rho_{n-1}\right\}\right)$ is a string C-group, then $n=3$. that

We may translate this theorem in abstract regular polytopes theory. It means

- if $S z(q)<G \leq \operatorname{Aut}(S z(q))$, then $G$ is not the automorphism group of an abstract regular polytope;

- if $G=S z(q)$, there exists an abstract regular polytope $\mathcal{P}$ such that $G=$ $\operatorname{Aut}(\mathcal{P})$. Moreover, if $\mathcal{P}$ is an abstract regular polytope such that $G=$ $\operatorname{Aut}(\mathcal{P})$, then $\mathcal{P}$ must be an abstract polyhedron, i.e. a rank three polytope.

\section{Almost simple groups of Suzuki type and C-Groups}

We first recall an easy lemma which will be used in the proof of Theorem 1 , The proof is left to the reader.

Lemma 1. Let $G$ be a group and let $H$ be a proper subgroup of $G$ such that all involutions of $G$ are in $H$. Then $G$ is not a C-group.

Obviously, if a group is not a C-group, then it is not a string C-group, and therefore it does not act regularly on an abstract polytope.

Lemma 2. Let $S z(q)<G \leq \operatorname{Aut}(S z(q))$ with $q \neq 2$ an odd power of two. Then $G$ is not a C-group.

Proof. Let $q=2^{2 e+1}$ with $e>0$ an integer. Since $\operatorname{Aut}(S z(q)) \cong S z(q): C_{2 e+1}$ where $C_{2 e+1}$ denotes a cyclic group of order $2 e+1$, and since $2 e+1$ is odd, the groups $S z(q)$ and $\operatorname{Aut}(S z(q))$ have the same number of involutions. Therefore, applying Lemma 1, we conclude that $G$ is not a C-group.

Lemma 3. Let $G=S z(q)$ with $q \neq 2$ an odd power of two. There exists a set $\left\{\rho_{0}, \rho_{1}, \rho_{2}\right\}$ of involutions of $G$ such that $\left(G,\left\{\rho_{0}, \rho_{1}, \rho_{2}\right\}\right)$ is a string C-group.

Proof. We may assume that $G$ is a permutation group acting two-transitively on a set $\Omega$ of $q^{2}+1$ points. Take two involutions $\rho_{0}, \rho_{1} \in G$ such that $\left\langle\rho_{0}, \rho_{1}\right\rangle=$ $D_{2(q-1)}$, where $D_{2(q-1)}$ denotes a dihedral group of order $2(q-1)$. Each of these two involutions fixes exactly one point of $\Omega$. Let $p_{i} \in \Omega$ be the point fixed by $\rho_{i}$ $(i=0,1)$. It is well known that $p_{0} \neq p_{1}$. Take an involution $\rho_{2} \in G$ such that $\rho_{2} \in G_{p_{0}}$. Then $\left\langle\rho_{0}, \rho_{2}\right\rangle=D_{2 n}$ for some $n$, and since $\rho_{0}$ and $\rho_{2}$ both fix the same point of $\Omega$, we have $n=2$. Moreover, we have $\left\langle\rho_{1}, \rho_{2}\right\rangle=D_{2 m}$ for some $m$ with $m \neq 2$ and the order of $\rho_{1} \rho_{2}$ is $m$. The group $G=\left\langle\rho_{0}, \rho_{1}, \rho_{2}\right\rangle$ is thus a string C-group. The corresponding polyhedron is of type $\{q-1, m\}$. The number $m$ depends on the choice of $\rho_{2}$.

Lemma 4. Let $\left(G,\left\{\rho_{0}, \ldots, \rho_{n-1}\right\}\right)$ be a string $C$-group such that $G \cong S z(q)$ with $q \neq 2$ an odd power of two. Then $n=3$. 
Proof. We have $n>2$, for if $n=2$, the group $G$ must be a dihedral group. Suppose that $n>3$. Then, $G$ possesses a subgroup $H=\left\langle\rho_{0}, \rho_{1}, \rho_{3}, \ldots, \rho_{n-1}\right\rangle \cong$ $\left\langle\rho_{0}, \rho_{1}\right\rangle \times\left\langle\rho_{3}, \ldots, \rho_{n-1}\right\rangle=D_{2 m} \times K$, where $D_{2 m}=\left\langle\rho_{0}, \rho_{1}\right\rangle$ and $K=\left\langle\rho_{3}, \ldots, \rho_{n-1}\right\rangle$. Looking at the maximal subgroups of $S z(q)$ as given for instance by Michio Suzuki in [3], we readily see that the only subgroups of the form $D_{2 m} \times K$ in $S z(q)$ are with $m=2$. If $H=D_{2 m} \times K=2^{2} \times K$, then $G=\left\langle H, \rho_{2}\right\rangle$, and the subgroup $\left\langle\rho_{0}\right\rangle$ is thus a normal subgroup of $G$, a contradiction. Therefore, $n=3$.

Proof of Theorem 1. The proof is obtained by putting together Lemmas 2, 3and4,

\section{REFERENCES}

[1] D. Leemans and L. Vauthier. An atlas of abstract regular polytopes for small groups. Aequationes Math., to appear.

[2] P. McMullen and E. Schulte. Abstract regular polytopes, volume 92 of Encyclopedia of Mathematics and its Applications. Cambridge University Press, Cambridge, 2002. MR1965665 (2004a:52020)

[3] M. Suzuki. On a class of doubly transitive groups. Ann. of Math., 75:105-145, 1962. MR0136646(25:112)

Département de Mathématiques, Université Libre de Bruxelles, C.P.216 - Géométrie, Boulevard du Triomphe, 1050 Bruxelles, Belgium

E-mail address: dleemans@ulb.ac.be 